\title{
Novel Emergency Department High Utilizer Surveillance In New Hampshire
}

\author{
David J. Swenson*1, Cole Zanetti ${ }^{2}$, Elizabeth R. Daly ${ }^{1}$ and Jose T. Montero ${ }^{1}$ \\ ${ }^{1}$ State of $\mathrm{NH}$, Concord, NH, USA; ${ }^{2} \mathrm{NH}$ Dartmouth Family Medicine Residency, Concord, NH, USA
}

\section{Objective}

To develop a manageable surveillance methodology to detect Emergency Department (ED) patients with the highest healthcare utilization, and monitor their targeted treatment improvement and medical health cost reductions over time for overall improvements in statewide health.

\section{Introduction}

Researchers have demonstrated benefits to identifying and developing interventions for patients that frequently seek healthcare services in the ED. The New Yorker Magazine, recently published an article titled The Hot Spotters, summarizing work being done in the United States to lower medical costs by giving the neediest patients better healthcare (1). In Camden, NJ, Physician Jeffrey Brenner closed his regular practice to focus on Hot Spotter patients (directing resources and brainpower to help their improvement) and measured a 40\% reduction in hospital inpatient and ED visits and a 56\% medical cost reduction for the first 36 Hot Spotters. A 2008 NH Office of Medicaid Business and Policy (OMBP) outpatient Medicaid ED frequency visit study was conducted, which cited that frequent ED users were more likely to have higher costs and rates of illness or disease than all Medicaid members (2). It was noted that increased prevention and wellness could reduce frequent ED use and increase cost savings ( $5 \%$ of the $\mathrm{NH}$ Medicaid population contributed to approximately $38 \%$ of ED costs). The NH Division of Public Health Services initiated a pilot project to examine NH Emergency Department (ED) surveillance data to identify high utilizer patients and realize improved health benefits and medical cost reductions.

\section{Methods}

This pilot focused first on identifying 2010 ED high utilizers in one hospital. A high utilizer was defined as a patient with 9 or more ED visits (top 10\%) and were identified using Medical Record Number followed by sorting number of visits in descending order. Visit diagnosis codes were identified for the top $10 \%$ of high utilizers, along with the date of admission, resident town, age, and gender. Finally, the top $10 \%$ over utilizers were mapped, identifying the total number of utilzers in each town. We overlaid this information on a map of NH's social vulnerability index to provide comparative data. Plans for future expansion include: Creating a GIS system that trends data over 10 years; Identifying ED high utilizers by location (visits by town, number of diagnosis codes per visit, date of visits, and summative data); and Creating hospital and total state-wide breakdown of highest utilizers, most common state-wide diagnosis for ED high utilizers, and specific data from each hospital in relation to state-wide social vulnerability index data.

\section{Results}

Over 56,000 ED visits at one pilot hospital were analyzed targeting 2,005 high utilizer visits for 158 patients. Identification by location, age, gender and ICD-9-CM diagnosis coding was determined for each ED over utilizer. Mapping of the high utilizers showed that 142 of the 158 patients resided in the city where the hospital was located. The most frequent diagnosis codes for the top 10\% ED high utilizers included mental health, substance abuse, and chronic disease conditions (such as diabetes mellitus).

\section{Conclusions}

This pilot project provides a manageable approach for identifying ED high utilizers for both health improvement and cost reduction surveillance efficiencies, and is a model for other surveillance management monitoring applications. Potentially, 2.1 million dollars per year could be saved, if each over utilizer used the ED one less time (2).

\section{Keywords}

Surveillance; Informatics; Chronic Disease

\section{References}

1. The Hot Spotters, Can we lower medical costs by giving the neediest patients better care? Gawande A, The New Yorker, Medical Report, January 24,2011

2. Office Of Medicaid Business And Policy, NH Comprehensive Health Care Information System (CHIS). Frequent Outpatient Emergency Department Use by New Hampshire Medicaid Members (An Evaluation of Prevalence, Diagnoses, Utilization, and Payments). Concord; October 2008.

*David J. Swenson

E-mail: dswenson@dhhs.state.nh.us 